\title{
Neurophysiological evidence that perceptions of fluency produce mere exposure effects
}

\author{
P. Andrew Leynes ${ }^{1} \cdot$ Richard J. Addante ${ }^{2}$
}

Published online: 22 April 2016

(C) Psychonomic Society, Inc. 2016

\begin{abstract}
Recent exposure to people or objects increases liking ratings, the "mere exposure effect" (Zajonc in American Psychologist, 35, 117-123, 1968), and an increase in processing fluency has been identified as a potential mechanism for producing this effect. This fluency hypothesis was directly tested by altering the trial-by-trial image clarity (i.e., fluency) while Event-Related Potentials (ERPs) were recorded. In Experiment 1, clarity was altered across two trial blocks that each had homogenous trial-by-trial clarity, whereas clarity varied randomly across trials in Experiment 2. Blocking or randomizing image clarity across trials was expected to produce different levels of relative fluency and alter mere exposure effects. The mere exposure effect (i.e., old products liked more than new products) was observed when stimulus clarity remained constant across trials, and clear image ERPs were more positive than blurry image ERPs. Importantly, these patterns were reversed when clarity varied randomly across test trials, such that participants liked clear images more than blurry (i.e., no mere exposure effect) and clear image ERPs were more negative than blurry image ERPs. The findings provide direct experimental support from both behavioral and electrophysiological measures that, in some contexts, mere exposure is the product of top-down interpretations of fluency.
\end{abstract}

Keywords Fluency $\cdot$ Mere exposure $\cdot$ Event-related potentials

P. Andrew Leynes

leynes@tcnj.edu

1 Department of Psychology, The College of New Jersey, P.O. Box 7718, Ewing, NJ 08628-0718, USA

2 Department of Psychology, California State University, San Bernardino, San Bernardino, CA, USA

\section{Introduction}

Fluency, the ease of processing a stimulus, is a perceptual experience that influences recognition memory judgments (Whittlesea \& Williams, 2001a, 2001b) and judgments of learning (Mueller, Tuaber, \& Dunlosky, 2013; Rhodes \& Castel, 2008; but see Mueller, Dunlosky, Tauber, \& Rhodes, 2014). There is considerable evidence that fluency also affects a number of other social-cognitive judgments that draw upon memory processes to varying degrees, such as judgments of beauty (e.g., Reber, Schwarz, \& Winkielman, 2004), truth (e.g., Reber \& Schwarz, 1999), fame (e.g., Jacoby, Kelley, Brown, \& Jasechko, 1989), and affective decisions (e.g., Bornstein, 1989).

Memory studies commonly use masked-repetition priming methods to increase fluency, a procedure which uses memory (repetition) to speed perception (e.g., Jacoby \& Dallas, 1981). However, perceptual fluency has also been manipulated in other ways by varying the clarity of stimuli (Whittlesea, Jacoby, \& Girard, 1990), the foreground/background color combinations (e.g., Reber \& Schwarz, 1999), font (e.g., Westerman, Miller, \& Lloyd, 2003), and pre-experimental experience (e.g., comparing famous and non-famous faces; Nessler, Mecklinger, \& Penney, 2005).

Results from paradigms that repeat stimuli and those that alter perceptual features in order to change fluency each suggest that bottom-up processing influences fluency-related behavior (i.e., the effects result from stimulus properties). However, there is also mounting evidence that fluency effects upon recognition are the product of an interpretation of the perceptual information (i.e., top-down processing; e.g., Whittlesea \& Williams, 2001a, 2001b; but see Cleary, Morris, \& Langley, 2007; Karpicke, McCabe, \& Roediger, 2008 for important qualifications) rather than being driven merely by the physical properties of the stimuli. Strong evidence for the top-down account has emerged from studies that 
observed changes in recognition performance despite having no study list at all (i.e., counterfeit lists; Lloyd, Westerman, \& Miller, 2003; Westerman, Lloyd, \& Miller, 2002) and from studies that observed differences in memory performance across testing contexts that used identical physical stimuli. For example, increasing the number of repetition-primed trials produces a smaller effect on recognition (Westerman, 2008), and electrophysiology provided evidence that recognition memory processes can be affected by fluency variations on a trial-to-trial basis (Leynes \& Zish, 2012). These results suggest that the physical attributes of stimuli are not as important as the testing context.

\section{Fluency in recognition and mere-exposure effects}

All theoretical models of recognition memory acknowledge that memory traces vary in strength, such that some judgments are based on remembering specific details of the past (referred to as recollection), whereas other memory decisions are based on a sense of oldness (i.e., familiarity). While models differ with respect to the underlying characterization of these stronger memory traces (e.g., Parks \& Yonelinas, 2007; Wixted, 2007), they agree that impoverished memory traces can lead to recognition based on fluency (e.g., Leynes \& Zish, 2012; Whittlesea \& Leboe, 2003). Similarly, fluency can also influence social-cognitive judgments that draw upon weak memory traces. One such example is the "mere exposure effect," which refers to the observation that affective "liking" ratings for a stimulus increase following a brief exposure to it (Zajonc, 1968). Mere exposure has been found to be both a salient and a relevant factor across a wide range of social contexts, such as race (e.g., Zebrowitz, White, \& Wieneke, 2008), marketing for consumer choices (e.g., Morgenstern, Isensee, \& Hanewinkel, 2013; Tom, Nelson, Srzentic, \& King, 2007), and group attitudes (e.g., Crisp, Hutter, \& Young, 2009), but the mechanisms for this phenomenon remain poorly understood.

Theoretical explanations of the mere-exposure effect differ with respect to how fluency influences affective judgments. Some argue that the stimuli influence affective judgments without a "cognitive appraisal" (Zajonc, 2001, p.226) or that "the mere-exposure effect is neither conceptually nor perceptually driven" but rather affected by "implicit priming" (Ladd, Toscano, Cowings, \& Gabrieli, 2014, p. 176). These accounts imply that mere-exposure is the result of bottom-up or datadriven processing. Other explanations contend that the stimulus features (i.e., fluency) is interpreted (via a top-down process) and attributed to an emotional reaction when mereexposure effects are present (Bornstein, 1989; Dechêne, Stahl, Hansen, \& Wänke, 2009; Reber, Winkielman, \& Schwarz, 1998; Whittlesea \& Price, 2001). These varying accounts differ in a substantive way and have yet to be reconciled; therefore, novel studies that apply convergent methods can inform this debate.

The majority of the top-down evidence for mere exposure comes from studies using stimulus repetition as a method to increase both liking ratings (mere exposure) and recognition. Jacoby and Dallas (1981) argued that fluency serves as one marker of memory because people learn that stimuli are perceived easier after the first exposure. Unfortunately, such repetition priming methods might artificially accentuate the linkage between memory and mere exposure, as it importantly confounds two possible sources of fluency: repetition fluency (a memory signal) and perceptual fluency (a sensory signal). While these two sources of fluency are indeed often conflated in our real-world experiences, this common laboratory manipulation limits our ability to isolate fluency effects on affective judgments that are naturally independent of prior experience. Our theoretical understanding of mere-exposure effects can, therefore, be advanced by approaches using experimental designs that manipulate perceptual fluency independently of stimulus repetition and that collect concurrent electrophysiological (event-related potential, ERP) measures of cognitive processing.

\section{Even-related potentials (ERPs) and fluency}

ERPs are physiological measures of electrical brain activity (EEG) that are recorded from multiple locations across the scalp, time-locked to the presentation of a stimulus, and averaged across trials to reveal electrophysiological correlates of a given cognitive process or task condition. Observing different spatial and temporal patterns of ERPs are evidence for different cognitive processing between conditions (Rugg \& Coles, 1995). For example, different ERP components have been correlated with familiarity-based processing (i.e., old item ERPs are more positive than new ERPs at frontal-central electrodes peaking at about $400 \mathrm{~ms}$, often called the "FN400" effect; Curran, 2000) and recollection-based processing (i.e., old ERPs more positive than new at parietal electrodes peaking about $600 \mathrm{~ms}$, called the "parietal old/new effect" or "Late Positive Component", LPC) during recognition (e.g., for review see Rugg \& Curran, 2007).

Using similar logic, Leynes and Zish (2012) provided evidence of an ERP correlate of fluency that was dissociated from these other ERP components associated with conscious memory processes. In that study, participants studied words, but perceptual fluency was manipulated at test by presenting some words in a slightly blurry form (i.e., to reduce fluency). Visual clarity (i.e., clarity hereafter) was held constant from trial to trial in a block of either clear or visually blurred test probes in one experiment, whereas clarity varied randomly across trials in a second experiment. This design revealed the effects of clarity independent of repetition (blocked format) and the effects of random variations of clarity and repetition (random format). ERPs were then sorted and averaged 
according to the type of item (i.e., old or new) in order to examine repetition/recognition processing. Several key findings emerged from this study.

First, the typical familiarity-related, mid-frontal ERP effect (i.e., FN400) was observed when both repetition and clarity varied randomly across trials. In contrast, the blocked condition elicited a reliably different ERP pattern than the FN400: old item ERPs were more negative than new ERPs at posterior electrodes ( $\sim 300 \mathrm{~ms})$. A similar ERP effect has been reported during recognition tests of impoverished memories using a range of stimuli including kaleidoscope images (Voss \& Paller, 2009, 2010), pictographic characters (Wang, Li, Gao, Xiao, \& Guo, 2015), and fragments of words (Ryals, Yadon, Nomi, \& Cleary, 2011). The ERP component in these previous studies was hypothesized to reflect implicit memory contributions to recognition (Voss \& Paller, 2009, 2010; Ryals et al., 2011) or perceptual fluency (Wang, et al., 2015).

Second, Leynes and Zish (2012) found that both the behavioral responses and event-related potential (ERP) measures differed across the blocked and random test contexts, thereby demonstrating that relative fluency variations across test trials was affecting recognition. The authors argued that only repetition fluency (i.e., from old vs. new words) was varying across trials in the blocked clarity test because clarity was held constant across individual trials in this experiment. On the other hand, two different forms of fluency (i.e., clarity and repetition) varied across trials during the random clarity test. These testing situations produced different ERP components. Familiarity-based recognition was observed when clarity varied randomly (i.e., FN400), whereas the earlier negative posterior effects marked fluency-based recognition (from repetition) when clarity was held constant across trials.

Third, Leynes and Zish (2012) isolated physiological effects of visual clarity by contrasting clear and blurry ERPs elicited by new items. Comparing new words critically removes the influence of repetition fluency because new words are not repeated; therefore, visual clarity provides the only source of fluency across these two types of words. Clear and blurry ERPs differed during the blocked test (when recognition was supported by fluency); however, they did not differ during the random test (when recognition was supported by familiarity). Critically, the clarity ERP differences (clear vs. blurry) were remarkably similar to the repetition (old vs. new) ERP differences that marked fluency-based recognition during the blocked test. Taken together, the repetition (old vs. new) and clarity ERP effects provided evidence that (a) fluency can support recognition (Whittlesea \& Leboe, 2003) and that (b) fluency is correlated with a different ERP than familiarity (i.e., fluency ERP is characterized by old $<$ new at posterior electrodes at approximately $300 \mathrm{~ms}$, whereas the FN400 effect is characterized by old $>$ new at mid-frontal electrodes during approximately the same time, e.g.,300-500 ms).

\section{Study goals}

One way to understand the cognitive mechanisms that influence social-cognitive judgments of mere-exposure effects is to adapt the Leynes and Zish (2012) paradigm to include affective liking judgments of stimuli. Manipulating perceptual fluency independently of stimulus repetition - and recording ERPs - has the ability to adjudicate between the two conflicting theoretical accounts of mere exposure noted earlier. One explanation of mere-exposure effects contends that fluency is a bottom-up (e.g., data driven) process (e.g., Ladd et al., 2014; Zajonc, 2001) that leads subjects to endorse stimuli with more positive affective responses. Alternative accounts argue that mere exposure effects instead result from an interpretation of fluency that operates in a top-down fashion that considers stimulus features in context (e.g., Dechêne, Stahl, Hansen, \& Wänke, 2009; Whittlesea \& Price, 2001). One goal of the two experiments reported here is to resolve some of the apparent discrepancy between these accounts by using physiological measures to determine whether bottom-up or top-down processes are more crucial for mere exposure.

Therefore, the Leynes and Zish (2012) paradigm was adapted to investigate the mere exposure effect by having participants study images of novel consumer products in order to eliminate the possibility of any pre-experimental fluency that would accrue from prior exposure to the products (Nessler et al., 2005; Whittlesea \& Leboe, 2003). These novel images were presented rapidly during encoding in order to effectively remove the role that recollection may contribute to recognition judgments (Whittlesea \& Price, 2001). This key factor reduces the possibility that elaborative recollection processing is contributing to any observed potential ERP differences. Following Leynes and Zish, the experiments differed only in the trial sequences. Image clarity (clear/blurry) was blocked in Experiment 1 so that only repetition fluency varied across trials. Alternatively, both clarity and repetition fluency varied randomly across trials in Experiment 2. During both tests, ERPs were recorded while participants made recognition (old or new) decisions followed by "liking" judgments for all probes. The additional "liking" judgment measured mundane affective responses that might be subject to influences from prior perceptual experiences (i.e., mere exposure), and is a key innovation of the current work from the Leynes and Zish study that investigated recognition.

\section{Study predictions}

In the present studies, perceptual fluency is manipulated independently of repetition, such that the bottom-up perceptual characteristics of the stimuli are identical across the two tests - with only the trial sequence of clarity altered. The ERP contrasts (old vs. new; clear vs. blurry) uncover novel 
evidence that can provide important insight into the mechanisms responsible for mere-exposure effects. For example, a similar pattern of ERP results for repetition (i.e., old vs. new) and clarity (i.e., clear vs. blurry) across the two different testing contexts of random and blocked clarity sequences would support accounts of bottom-up influences on affective judgments (e.g., Zajonc, 2001; Ladd et al., 2014). Such results would indicate that the perceptual qualities of the stimuli or implicit memory are driving the affective judgments. Alternatively, ERP differences across these two experiments would be important evidence that fluency is the product of a top-down interpretive mechanism (e.g., Bornstein, 1989; Reber et al., 1998; Whittlesea \& Price, 2001) and that fluency is evaluated relative to recent events (i.e., on a trial-by-trial basis). Furthermore, if the ERP patterns are similar to those reported during fluency-based recognition (e.g., Leynes \& Zish, 2012), the ERP evidence will support a common mechanism for both recognition and mere-exposure.

\section{Method}

\section{Participants}

All participants were College of New Jersey undergraduate students (aged 18-21 years old) who participated for a partial course requirement. A separate sample of participants were tested in each experiment (Exp. 1, N = 36, 11 males; Exp. 2, $\mathrm{N}=33$, four males). All participants were right-handed, had no history of neurological disorders, and reported that they were not using any form of psychotropic medication at the time of study. A monetary incentive of $\$ 25$ was offered to the participant with the fastest and most accurate responses on the recognition test in each sample.

\section{Stimuli}

Three hundred off-brand products were obtained through the use of internet search engines. The products included a variety of common objects available in supermarkets and/or big box retail stores. The top of Fig. 1 provides examples of stimuli that were used in both experiments. In order to eliminate pre-experimental familiarity and liking, stimuli were constrained to novel, offbrand products as determined by a pilot test on a separate sample of participants. Each product image was edited to have a black background, and images fit the entire screen during both experiments. Images that were slightly blurrier than their clear counterparts (bottom of Fig. 1) were created following the Leynes and Zish (2012) procedures.

\section{Procedure}

Each participant completed two tasks: a shallow encoding task and a recognition test (i.e., Old/New discrimination). During the encoding phase, participants viewed a series of clear pictures (150 trials) that were presented one at a time on the computer screen for $100 \mathrm{~ms}$ followed by a black screen for $100 \mathrm{~ms}$ before the next picture was presented. This rapid serial visual presentation was used to create impoverished memory traces that would be unlikely to support recollection (Whittlesea \& Price, 2001).

The recognition test consisted of 300 product images, half of which were old items from the encoding task and half of which were blurred creating 75 items of each type (e.g., old clear, old blurry, new clear, and new blurry). The computer software randomized the order of old and new images for each participant. During Experiment 1, clarity was not varied on a trial-by-trial basis; rather, it was varied across trial blocks (one block of 150 clear products and one block of 150 blurry products, counterbalanced across participants). During Experiment 2, clarity and study status (i.e., old or new) varied on a trial-bytrial basis according to a random trial sequence created by the software.

On each test-trial, participants were asked to look at images in the center of the computer screen and then to judge whether the image was previously encountered in the first task (i.e., "old") or whether the image was "new." Participants made a simultaneous recognition and confidence judgment using marked keyboard keys that corresponded to a six-point Likert Scale (1 = "Sure New", 2 = "Moderately Sure New", 3 = "Less Sure New", 4 = "Less Sure Old", 5 = "Moderately Sure Old", 6 = "Sure Old"). The product image remained on the screen until a response was given and then the screen was cleared for $1.5 \mathrm{~s}$.

Next, the computer displayed a prompt for a liking rating of the preceding image. Participants indicated product opinions by pressing one of six marked keys that corresponded to a sixpoint Likert scale ( 1 = "Strongly Dislike", 2 = "Dislike", 3 = "Somewhat Dislike", 4 = "Somewhat Like", 5 = "Like", 6 = "Strongly Like"). The screen cleared and the computer automatically advanced to the next trial after a response. Participants were encouraged to respond as quickly and as accurately as possible on the test.

Post-task Before a formal debriefing of the purpose of the experiment, participants responded to three questions in an effort to assess the participant's awareness of the fluency changes during the memory test. The experimenter first asked participants "What do you think was the purpose of the study?" followed by "Have you used or seen any of the products before? If so, which one?" and finally "Did you notice anything else about the pictures? If so, what did you notice?" The experimenter recorded the responses on a sheet of paper. 
Study Phase (only clear pictures)

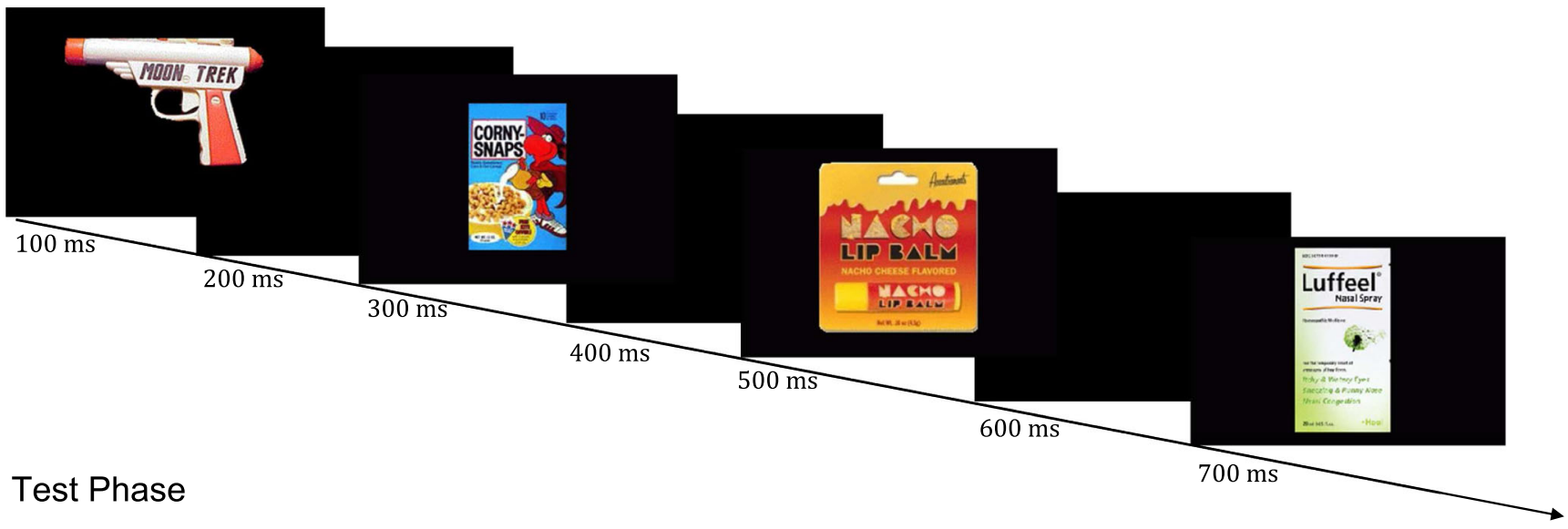

Old Items

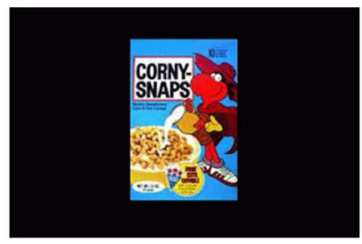

Clear

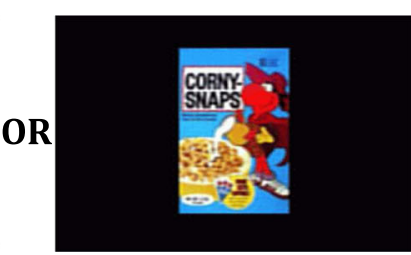

Blurry

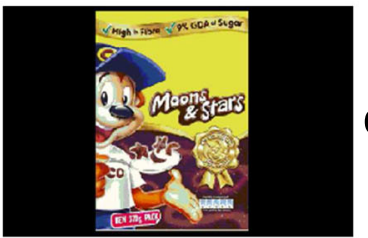

Clear

New Items

Fig. 1 Each product was presented for $100 \mathrm{~ms}$ and followed by a black screen for another $100 \mathrm{~ms}$ (top) during encoding. During the test phase, products were presented in either a clear (same as study) or blurry version

In order to eliminate pre-experimental exposure effects, any images that participant's identified as having seen before the experiment were eliminated from the analyses (only 22 trials out of 10,500 total, $.002 \%$, across both experiments were eliminated).

ERP recording procedures and analysis $\mathrm{EEG}$ was sampled during the recognition test from $29 \mathrm{Ag} / \mathrm{AgCl}$ electrodes mounted in an elastic cap (Neuromedical Supplies, Inc.) referenced to the left mastoid. Electrodes were placed over the frontal lobes (Fp1, Fp2, F7, F3, Fz, F4, F8, FC3, FCz, FC4), temporal lobes (FT7, FT8, T7, T8, TP7, TP8), parietal lobes (CP3, CPz, CP4, P7, Pz, P4, P8), occipital lobes (O1, O2), and the central position of the scalp $(\mathrm{C} 3, \mathrm{Cz}, \mathrm{C} 4)$. An additional active electrode was attached to the right mastoid. The vertical electroculogram (vEOG) was recorded bipolarly using two $\mathrm{Ag} / \mathrm{AgCl}$ electrodes placed above and below the participant's left pupil. Horizontal electroculogram (hEOG) was recorded bipolarly from a pair of identical electrodes and attached to the outer canthi of both eyes. Interelectrode impedance was below $5 \Omega$. EEG and EOG signals were continuously sampled at a rate of $150 \mathrm{~Hz}$ using an amplifier $(0.03-40 \mathrm{~Hz}$ frequency range at $-3 \mathrm{~dB}$ attenuation). EEG data was not recorded during the encoding task.

The continuous data file was separated into $2,200 \mathrm{~ms}$ epochs beginning with the image onset to create epochs. Voltage was baseline corrected to the average activity
$300 \mathrm{~ms}$ prior to presentation of the test image, re-referenced to the average of the left and right mastoids, and digitally filtered using a $30-\mathrm{Hz}$ low pass filter $(-3 \mathrm{~dB} / \mathrm{oct})$. Ocular artifacts were corrected using the algorithm developed by Semlitsch, Anderer, Schuster, and Presslich (1986). After correcting for ocular artifacts, trials for which ERP amplitudes exceeded $\pm 150 \mu \mathrm{V}$ were excluded from the analyses by the software. In both experiments, omitted trials were equally distributed across the experimental conditions (Experiment 1: $M$ $=17 \%$, S.D. $=19 \%$; Experiment 2: $M=12 \%$, S.D. $=13 \%$ ).

ERPs elicited by accurate responses were averaged after artifact reduction to examine recognition ERP effects (i.e., old vs. new) collapsed across clear and blurry conditions yielding two ERPs for each experiment [Experiment 1: old ( $M=76$ trials, range 47-105), new $(M=94$, range 54-128); Experiment 2: old $(M=69$, range $50-90)$, new $(M=74$, range $46-130)$ ]. Next, they were averaged by collapsing across old and new items to create clear and blurry ERPs [Experiment 1: clear $M=77$ trials, range 40-106; blurry $M$ = 82, range 39-103); Experiment 2: clear $M=71$, range 5388, blurry $M=71$, range 46-108)].

ERP time windows targeted for analyses were taken from Leynes and Zish $(2012$; 180-220 ms, 280-400 ms, 400$600 \mathrm{~ms}$, and $900-1100 \mathrm{~ms}$ after image onset). These ERP time windows were analyzed at frontal ( $\mathrm{F} 3, \mathrm{Fz}, \mathrm{F} 4)$, frontal-central (FC3, FCz, FC4), central (C3, Cz, C4), central-parietal (CP3, $\mathrm{CPz}, \mathrm{CP} 4)$, and parietal electrodes (P3, Pz, P4). An ANOVA 
model that contained a within-subjects factor of Item Repetition (old, new), Clarity (clear, blurry), Anterior/ Posterior (AP) electrode location (5 levels front to back), and Left/Right hemisphere (LR) location (3 levels, left to right) analyzed the ERP amplitudes. ${ }^{1}$

\section{Results}

To facilitate an understanding of recognition (i.e., repetition) effects and visual clarity effects, the results of Experiment 1 and Experiment 2 are summarized together for the various dependent measures. The first section of the results reports recognition memory effects. Figure 2 displays the grandaverage ERPs elicited by correctly recognized old and new images (collapsed across clear and blurry) at the midline, frontal-central $(\mathrm{FCz})$, and parietal $(\mathrm{Pz})$ electrodes, to be consistent with studies from other laboratories (e.g., Voss \& Paller, 2010). The left-half of Fig. 2 displays the old-new ERP effects during Experiment 1 (blocked clarity across trials), whereas the right-half of the figure displays the old-new effects for Experiment 2 (random clarity variations across trials). The topographies of significant old-new effects are shown below the ERP traces during the 900-1,100-ms time interval. $^{2}$

\section{Recognition memory results}

\section{Experiment 1 - Blocked clarity}

\section{Recognition memory behavioral results}

Responses The top left of Table 1 displays the mean proportion of Hits (old products called "old") and Correct Rejections (CR; new products called "new"). These measures were analyzed using a repeated-measures ANOVA model with Item type (Hit/CR) and Mask (clear/blurry) as factors. Clear probes elicited more Hits and CRs than blurry probes (Mask: $F(1,35)$ $=8.31, p=.007)$, and there were more CRs than Hits overall

\footnotetext{
${ }^{1}$ We also performed two other ERP analyses. First, we adjusted the time intervals used to capture the FN400 (500-700 ms) and LPC (700$900 \mathrm{~ms}$ ) based on visual inspection of the ERP traces. Second, we averaged and analyzed ERPs based on liking ratings (like vs. dislike) rather than sorting and averaging based on clear/blurry or old/new differences. Neither the adjusted intervals analysis nor the liking ratings analyses yielded any findings relevant to the points addressed in the current article; consequently, we do not discuss these results any further.

${ }^{2}$ Topographic maps of earlier latencies traditionally reported for explicit memory studies (FN400: 400-600 ms and LPC: 600-900 ms) are not illustrated because those latencies exhibited no detectable differences between conditions. As reported in the results, the later latency of 900$1,100 \mathrm{~ms}$ was the only latency during which reliable old-new differences were detected.
}

(Item: $F(1,35)=14.26, p<.001)$. There was no significant interaction between Item $\times$ Mask: $F(1,35)<1, p>.05$.

Measures of responder sensitivity (d') and criterion (C) were calculated and analyzed for each mask type. Sensitivity was better when probes were clear $(M=.842, S . D .=.340)$ versus blurry $(M=.686, S . D .=.300 ; F(1,35)=7.06, p$ $=.012$ ). Both $\mathrm{d}^{\prime}$ were greater than a zero value (clear: $t(35)=$ $14.85, p<.001$; blurry: $t(35)=13.72, p<.001)$. Estimates of criterion did not differ as a function of mask (clear: $M=.158$, S.D. $=.244$; blurry: $M=.131$, S.D. $=.339 ; F(1,35)<1)$; however, both criterion estimates were greater than a zero value (clear: $t(35)=3.904, p<.001$; blurry: $t(35)=2.32, p$ $=.026$ ) indicating a conservative criterion was adopted during both tests.

Response times The median response times for correct recognition judgments are presented in Table 1 (middle, left rows). None of the effects were significant (Item: $F(1,35)=$ $1.45, p>.2$; Mask: $F(1,35)<1$; Item $\times$ Mask: $F(1,35)<1)$. Hence, response times were unaffected by the experimental manipulations.

Confidence ratings Average confidence ratings (bottom, left rows of Table 1) were greater for old products (Item: $F(1,35)$ $=39.18, p<.001$ ) and for clear images (Mask: $F(1,35)=5.67$, $p<.023)$. There was no significant interaction between Item $\times$ Mask: $F(1,35)<1$.

\section{Recognition memory ERP results}

The ERP recognition effects (i.e., Old/New collapsed across clear and blurry conditions) are presented in Fig. 2 (left-side). ERP differences were detected during the $600-800 \mathrm{~ms}$ (Item $\times$ AP: $F(2,120)=3.66, p<.001)$ and $900-1,100 \mathrm{~ms}$ time windows (Item: $F(1,35)=7.77, p=.009$; Item $\mathrm{x}$ AP: $F(1.48,51.8)=5.09, p=.028)$. The nature of this difference was that the old ERPs were more negative than new ERPs, which is a reversal of the typical LPC effect that might have been otherwise expected if a longer encoding exposure had been used (Bruett \& Leynes, 2015). This memory-related difference was maximal at the left-midfrontal electrodes.

\section{Experiment 2 - Random clarity}

\section{Recognition memory behavioral results}

Responses Random changes in image clarity did not affect recognition (Table 1), Item: $F(1,32)=1.86, p>.05$. Mask: $F(1,32)<1, p>.05$, Item $\times$ Mask $F(1,32)=3.6, p>.05$. Furthermore, responder sensitivity did not differ as a function of visual clarity (clear: $M=.252$, S.D. $=.341$; blurry: $M=$ .229 , S.D. $=.374 ; F(1,32)<1)$. Both d' values were greater than zero (clear: $t(32)=4.24, p<.001$; blurry: $t(32)=3.516$, 


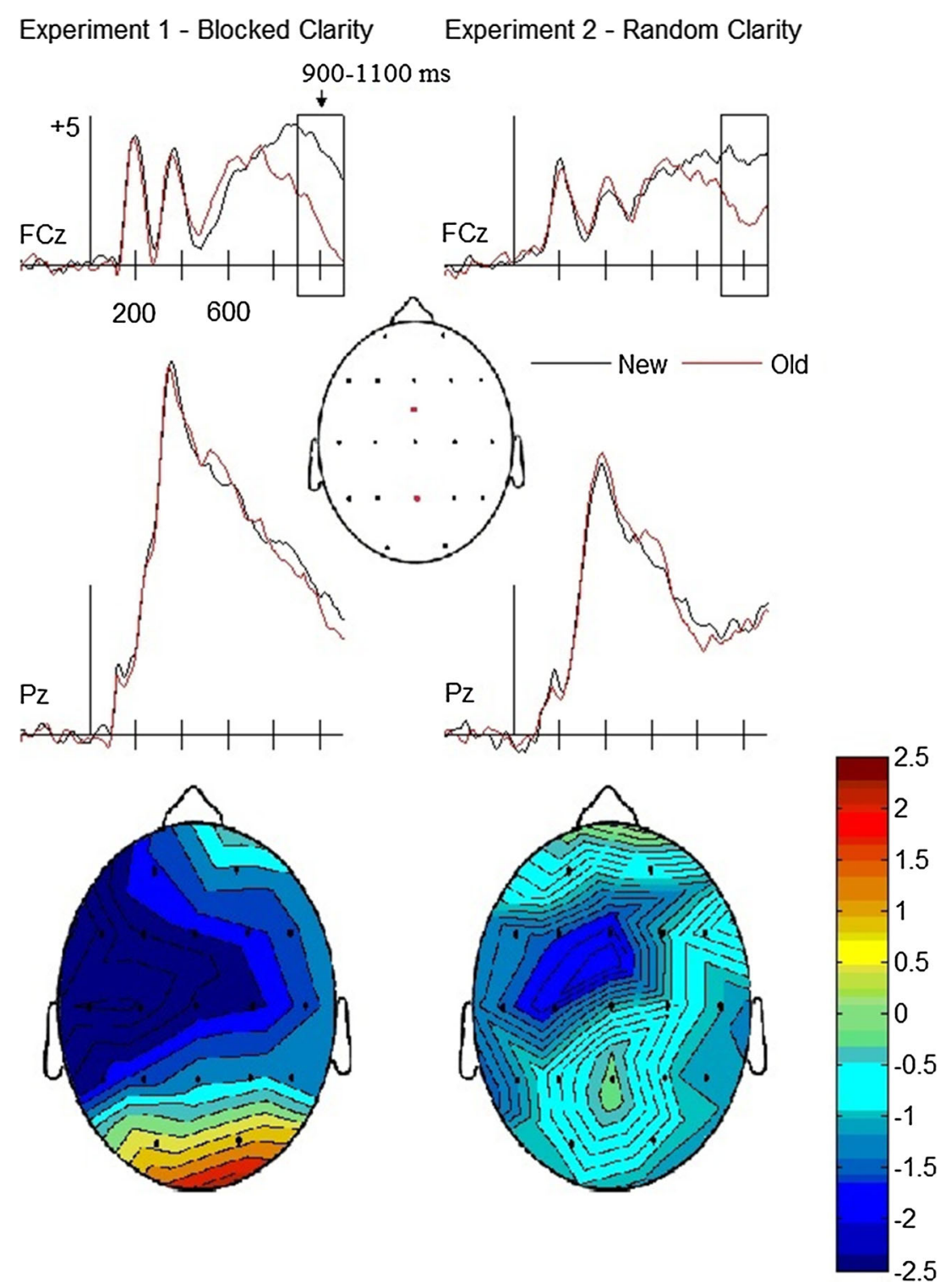

Fig. 2 Old/new event-related potentials (ERPs) depicting the reversed memory ERP effects (i.e., old $<$ new). The topographic maps plot the old-new ERP difference during the 900- to 1,100-ms interval

$p=.001)$. Estimates of criterion were marginally significant (clear: $M=.079$, S.D. $=.272$; blurry: $M=.009$, S.D. $=.218$; $F(1,32)=3.86, p=.058)$. Neither criterion estimate differed from a zero value ( $\mathrm{ps}>.1$ ), indicating a moderate criterion was adopted when viewing both clear and blurry probes.

Response times The RT analysis indicated that when clarity was random, response times for Hits significantly faster than CR RTs (Item: $F(1,32)=10.32, p=.003$ ). None of the other effects were significant, Mask: $F(1,32)=3.00, p>.05$, Item $\times$ Mask: $F(1,32)<1$.

Confidence ratings Average confidence ratings (right side of Table 1) did not vary between old and new products $(F(1,32)$ $<1)$; however, ratings varied as a function of Mask $(F(1,32)=$
$6.79, p=.014)$ and this effect was qualified by the significant Item $\times$ Mask interaction, $F(1,32)=7.40, \mathrm{p}=.01$. Post-hoc comparisons revealed that confidence did not vary for old items $(F(1,32)<1)$, whereas responses were more confident for clear, new items $(F(1,32)=8.72, \mathrm{p}=.006)$.

\section{Recognition memory ERP results}

The old/new ERP effects are depicted in Fig. 2 (right-side). There were no significant old/new ERP effects, except during the $900-1,100 \mathrm{~ms}$ interval (Item $\times \mathrm{AP} \times \mathrm{LR}: F(5.76,184.32)$ $=4.09, p=.001)$. The topographic map indicates that the "oldmore-negative-than-new" difference was maximal at leftmidfrontal electrodes, which was the same old-new difference and topography that was observed in Experiment 1. 
Table 1 Proportions of recognition responses, response times, and confidence ratings for Hits and Correct Rejections (CR) as a function of visual clarity for Blocked and Random presentation

\begin{tabular}{|c|c|c|c|c|}
\hline & \multicolumn{2}{|c|}{ Experiment 1 - Blocked Clarity } & \multicolumn{2}{|c|}{ Experiment 2 - Random Clarity } \\
\hline & Hits & $\mathrm{CR}$ & Hits & $\mathrm{CR}$ \\
\hline \multicolumn{5}{|l|}{ Mask } \\
\hline & \multicolumn{4}{|c|}{ Recognition responses } \\
\hline Clear & $.60(.09)$ & $.71(.12)$ & $.51(.10)$ & $.58(.14)$ \\
\hline \multirow[t]{2}{*}{ Blurry } & $.57(.13)$ & $.67(.13)$ & $.54(.09)$ & $.55(.12)$ \\
\hline & \multicolumn{4}{|c|}{ Response time (ms) } \\
\hline Clear & $2,421(912)$ & $2,495(906)$ & $2,471(773)$ & $2,826(1024)$ \\
\hline \multirow[t]{2}{*}{ Blurry } & $2,327(742)$ & $2,398(814)$ & $2,421(708)$ & $2,695(881)$ \\
\hline & \multicolumn{4}{|l|}{ Confidence } \\
\hline Clear & $2.31(.35)$ & $1.98(.41)$ & $1.85(.39)$ & $1.93(.39)$ \\
\hline Blurry & $2.19(.39)$ & $1.83(.48)$ & $1.87(.36)$ & $1.74(.54)$ \\
\hline
\end{tabular}

Note: Values in parentheses are the standard deviation

\section{Mere exposure effects}

The second section of the results focuses on mere-exposure effects by examining the behavioral measures of the mereexposure effect (i.e., liking ratings) and the electrophysiological contrast of clear and blurry product ERP differences. Figure 3 presents mere-exposure effects for Experiment 1 (left-half) and Experiment 2 (right-half). Panel A presents the average liking ratings as a function of repetition (old/new product) and clarity (clear/blurry). Inspection of this panel suggests that the traditional mere exposure effect was evident in Experiment 1 (old products were liked more than new) and that the direction of this effect was reversed in Experiment 2 (clear products were liked more than blurry). Panel B depicts the grand-average ERPs elicited by correctly recognized clear and blurry images (collapsed across old and new) in the same format as was presented in Fig. 2 for recognition effects. Panel $\mathrm{C}$ presents the scalp maps depicting the clear-blurry differences during each of the time intervals where significant differences were detected.

\section{Experiment 1 - Blocked}

\section{Mere exposure behavioral results}

Liking ratings The mere exposure effect was observed (Fig. 3, Panel A), in that old products elicited significantly greater liking ratings than new products (Item: $F(1,35)=5.50, p=.025$ ). The average liking ratings were not affected by image clarity (Clarity: $F(1,35)=1.70, p>.05$; Item $\times$ Clarity: $F(1,35)<1)$.

\section{Clarity ERP results}

Table 2 displays the ERP differences during each of the targeted time intervals. Clarity effects, characterized by clear products eliciting more positive ERPs than blurry products, were detected during each time interval (Fig. 3, Panel B). The topographic maps indicate that perceptual clarity-related ERP differences were largest at right-posterior electrode sites (Fig. 3, Panel C).

\section{Experiment 2 - Random}

\section{Mere exposure behavioral results}

Liking ratings The mere exposure effect was reversed from the pattern evident in Experiment 1 (Fig. 3, Panel A) because liking ratings were greater for clear than for blurry products $(F(1,32)=8.03, p=.008)$, but the liking ratings did not differ between old and new products (Item: $F(1,32)<1$; Item $\times$ Clarity, $F(1,32)<1)$.

\section{Clarity ERP results}

During Experiment 2, the perceptual clarity ERP effects observed in Experiment 1 were reversed (i.e., clear $<$ blurry) at several of the targeted ERP epochs (right half of Table 2; Fig. 3, Panel B). The topographies of these effects were more left-lateralized (Fig. 3, Panel C).

\section{Examination of clarity ERP effects in new products}

A major advantage of the current study is that ERPs elicited by new products in each of the two experiments represent perceptual fluency processing that is completely independent of differences due to repetition fluency (Leynes $\&$ Zish, 2012). Therefore, the ERPs elicited by new products were examined separately from those elicited by old items in order to isolate physiological effects related to 
Experiment 1 - Blocked Clarity

A) Liking Ratings

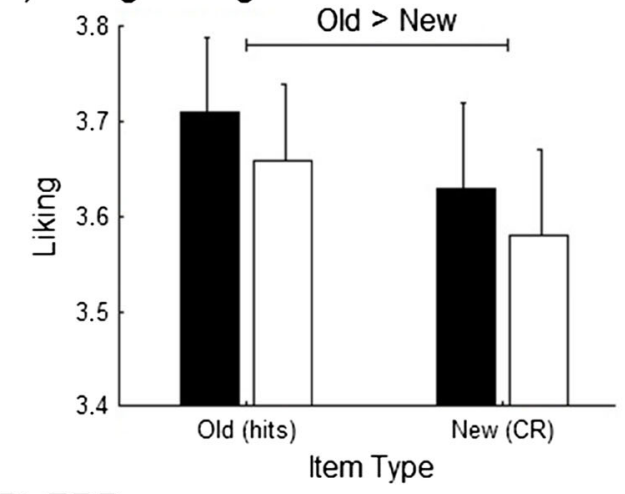

B) ERPs
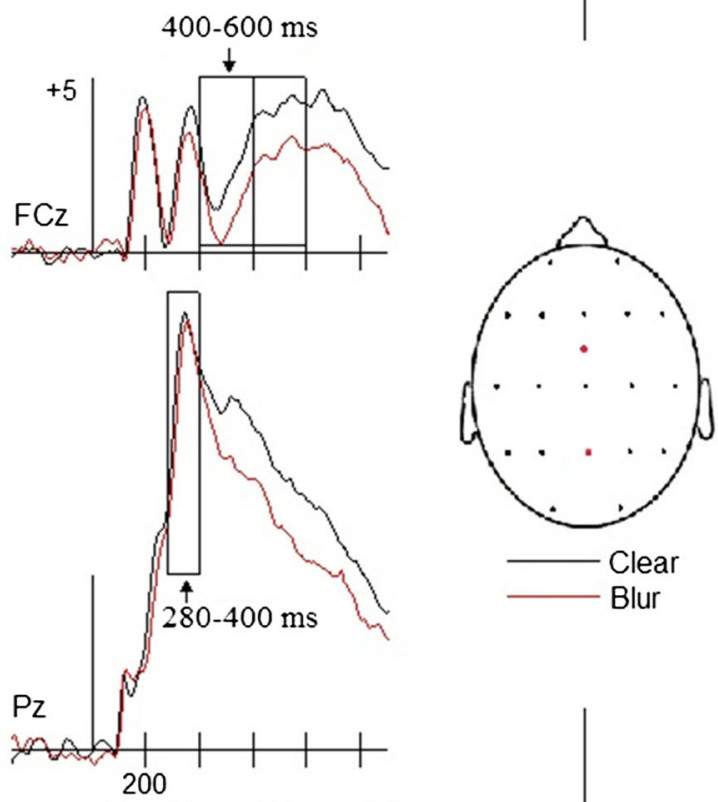

C) Topographic Clear-Blurry Maps

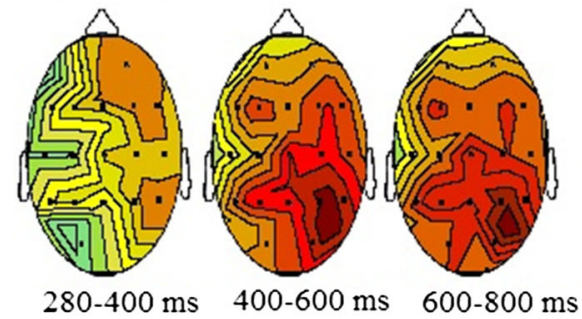

\section{Experiment 2 - Random Clarity}
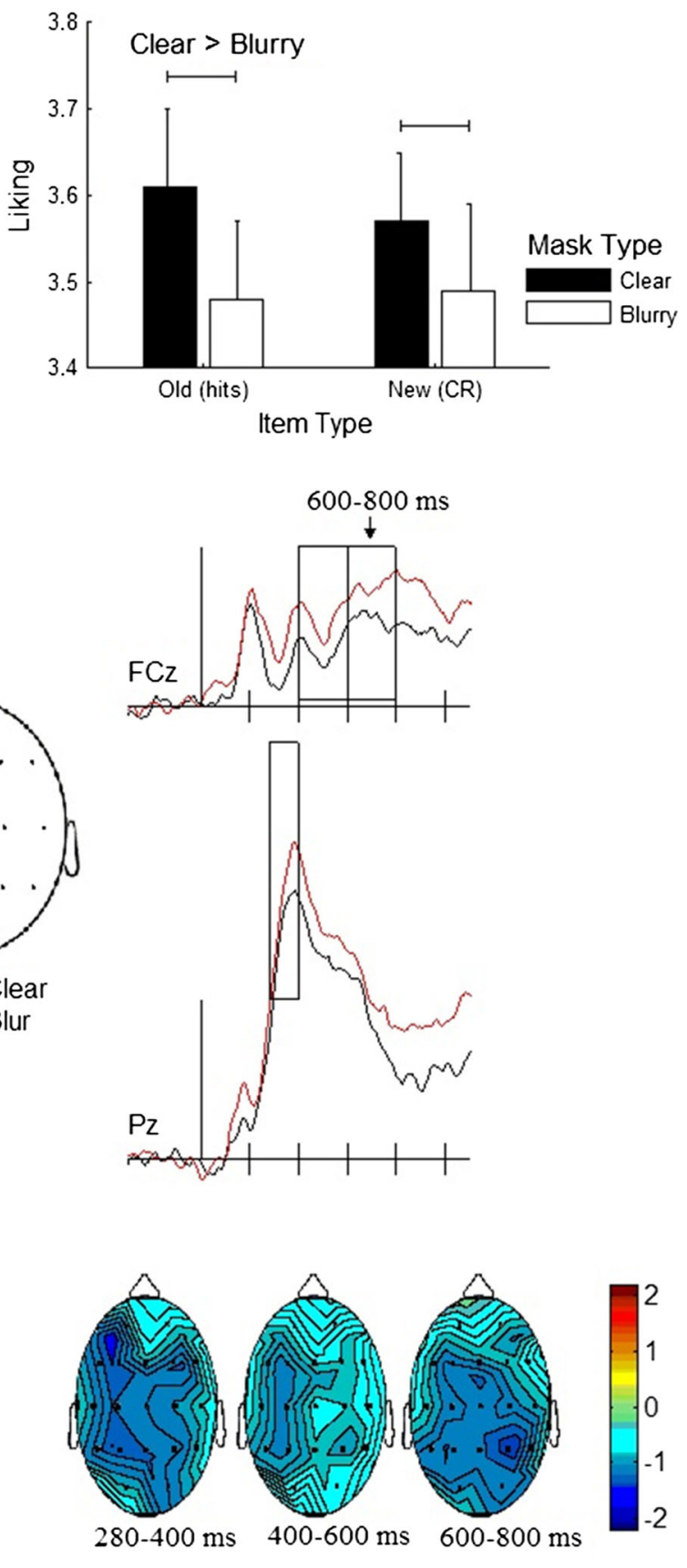

Fig. 3 Mean liking ratings (panel A), grand-average event-related potential (ERP) traces (panel B), and ERP topographic maps depicting the difference between clear and blurry ERPs as a function of blocked or random trial sequence

perceptual clarity, and provide insight into the physiological correlates supporting perceptual fluency. Figure 4 presents the grand-average ERPs elicited by new (right panel) and old (left panel) products for both of the experiments. Visual inspection of this figure suggests that the general patterns of perceptual ERP differences were similar for both old and new products regardless of the trial format.
To explore these effects, we conducted the same ERP analyses for each of these conditions separately. The results of these contrasts are presented in Table 2, in rows beneath each of the overall effects. In Experiment 1 (blocked clarity), the contrast of old products did not produce any significant differences related to perceptual clarity (all $p \mathrm{~s}>.05$ ); rather, the significant ERP effects were driven by neural activity for the 
Table 2 Fluency ERP contrasts (Clarity: clear vs. blurry) during blocked (Experiment 1) and random (Experiment 2) trial sequences

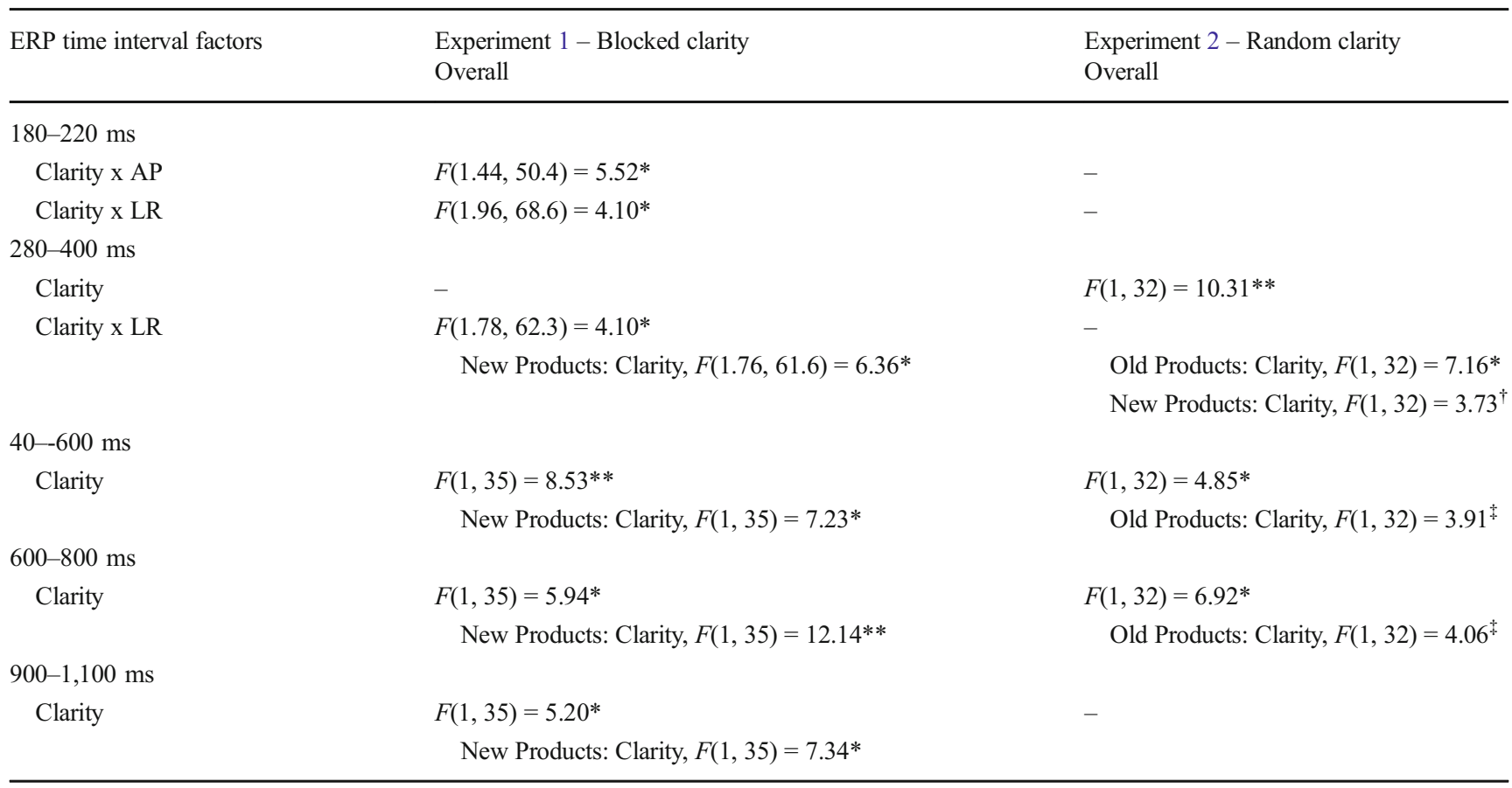

${ }^{\dagger} p=.062,{ }^{*} p<.058, * \mathrm{p}<.05, * * \mathrm{p}<.01$

$E R P$ event related potential, $A P$ anterior/posterior electrode location, $L R$ left/right electrode location

new products. In Experiment 2 (random clarity), the ERP effects of perceptual clarity were weaker (many were marginally significant) and were apparently driven by the old products because the analysis of ERPs elicited by new products produced only one marginally significant effect. These analyses provide evidence that the ERP patterns observed in both experiments are relatively independent of prior exposure; therefore, they are more reflective of a modulation of perceptual than recognition processing.

\section{Fluency manipulation awareness in both experiments}

The responses on the post-experimental questionnaire were evaluated to determine whether or not participants were aware of the fluency manipulation. No subjects were eliminated

\section{Experiment 1 - Blocked Clarity}
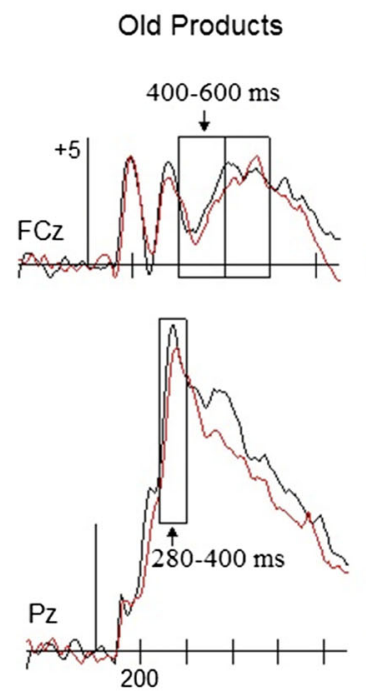

New Products

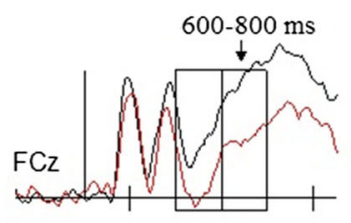

Experiment 2 - Random Clarity

Old Products

New Products
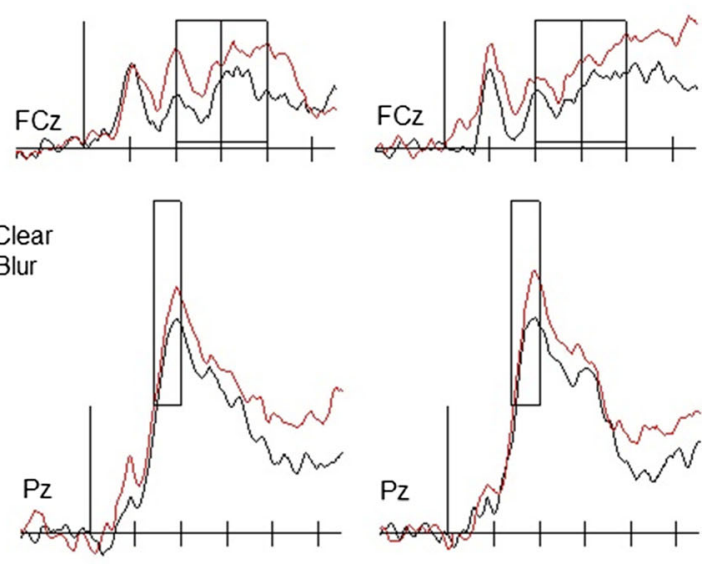

Fig. 4 Grand-average event-related potentials (ERPs) for Old and New Products as a function of presentation sequence 
from the sample based on their responses to these questions: questionnaire responses indicated that the purpose of the study was not evident to the participants, because only one participant (Experiment 1) was able to guess the exact purpose of the experiment (1/35 or .03\%). In fact, only a small portion of each experimental sample reported that some of the products were blurry when specifically asked about the stimuli (Exp. 1: $3 / 35$ or $.09 \%$; Exp. 2 : $6 / 33$ or $18.8 \%$ ). Consequently, it is unlikely that conscious awareness of perceptual changes between stimuli or conditions is contributing to the results.

\section{Discussion}

The current investigation manipulated perceptual fluency (i.e., visual clarity) and repetition in two separate experiments to measure their effects on liking ratings of commercial products (i.e., the mere exposure effect) and upon recognition judgments while brain potentials (ERPs) were recorded. Because repetition fluency and perceptual fluency were manipulated independently, the data were analyzed for recognition and clarity effects separately and these analyses revealed a number of key behavioral and ERP findings that have implications for recognition and mere-exposure. The implications of the findings on recognition are discussed first and are followed by a discussion of the implications for mere-exposure.

\section{Fluency and recognition}

Fluency had few effects on recognition in the current study both behaviorally and physiologically. Clear probes were recognized more than blurry probes when the sequence of trials was blocked (Exp. 1), but no differences in recognition were detected when the sequence of clear and blurry trials varied randomly (Exp. 2). The analyses of the recognition ERPs revealed the same pattern in both experiments: ERPs elicited by old products were more negative than new products beginning at approximately $600 \mathrm{~ms}$ (Exp. 1) and peaking during the 900-1,100 ms interval. These ERP differences were maximal at the left-midfrontal electrodes. Similar patterns have been previously reported when fluency supported recognition for words (Leynes \& Zish, 2012) and for low-confidence source judgments (Addante, Ranganath, \& Yonelinas, 2012), and in patients with hippocampal damage (Addante, 2015). Although the significance of this ERP pattern is currently unknown, the common thread across these studies is recognition judgments that are based upon impoverished information that might reflect a greater implicit memory contribution to an explicit memory task than is usually ascribed. Given that the current study procedures were designed to create impoverished memory traces (e.g., speeded encoding trials of novel images), such an account is consistent with these emerging results of implicit memory from other laboratories.

\section{Why would fluency alter affective judgments without affecting recognition?}

Whittlesea and Price (2001) reviewed the cognitivebehavioral literature on this issue, and they found that many studies observed fluency effects on liking but with no parallel effect on recognition. Here, we observed a similar pattern using physiological measures (ERP) of recognition. The typical recognition ERP components (i.e., FN400, LPC) were not observed during either test; therefore, these null recognition ERP findings are an important extension of the previously reported behavioral effects by using a physiological measure. Whittlesea and Price argued that fluency will be interpreted "back to consciousness as a value on whatever dimension was made salient by the task, causing a feeling of familiarity or pleasantness." (p. 244). Presumably, fluency in the present study was interpreted as liking because that judgment was more salient in this task; consequently, fluency did not cause feelings of familiarity and did not elicit the FN400 ERP component that has been repeatedly correlated with familiarity experiences (e.g., Rugg \& Curran, 2007).

The recognition ERP effects are even more salient, in this broad context, when compared to a recent similar ERP study of fluency and recognition. Bruett and Leynes (2015) manipulated product fluency by exposing subjects to name-brand (fluent) and novel, off-brand (non-fluent) products. Subjects made recognition and liking judgments - as in the two experiments reported in this article - during several different recognition tests that also manipulated trial-by-trial fluency levels. During one testing context, subjects viewed just products of one type of pre-experimental fluency (i.e., all name-brand or all off-brand products). This manipulation is analogous to the blocked clarity test used by Leynes and Zish (2012; and Exp. 1 of the present article) because repetition fluency was the only fluency varying across trials. On another test, both name- and off-brand products were presented in a random sequence. This context was analogous to random clarity test used by Leynes and Zish (Exp. 2 of the present article) because two sources of fluency (i.e., repetition and pre-experimental familiarity) were independently varying across test trials.

Several results reported by Bruett and Leynes (2015) are directly relevant to the interpretation of the results of the current experiments. First, the pattern of recognition ERP results of Bruett and Leynes were very similar to those reported by Leynes and Zish (2012); therefore, they provide an important conceptual replication and validation of the basic fluencyrelated ERP effects. Second, Bruett and Leynes also collected liking judgments, but they did not find any significant behavioral or ERP results related to this measure. Based on postexperiment interviews, subjects guessed that the purpose of the study was to examine how name-brand products were liked more than off-brand products. Consequently, the recognition component of the task was more salient than the affective 
judgments component because subjects had pre-conceived ideas about what they would like more. Therefore, the Bruett and Leynes test context prompted subjects to attribute fluency to recognition, whereas the context created in the present studies prompted subjects to attribute fluency to liking. Collectively, this evidence supports Whittlesea and Price's (2001) hypothesis that fluency is applied (via top-down processing) to whatever task component is more salient.

\section{Mere exposure and interpretations of fluency}

The prototypical mere-exposure effect was observed when visual clarity was held constant across trials (blocked trials), such that old (repeated) products were liked more than new products. The mere exposure effect was also associated with a distinct pattern of ERP differences, in which clear ERP amplitudes were more positive than blurry ERP amplitudes. Importantly, the behavioral mere-exposure effect reversed when visual clarity varied across trials, such that clear items were now liked more than blurry items and there was no difference in liking reported between old and new products. This behavioral reversal of liking ratings also corresponded with a reversed pattern of physiological differences as well, such that clear ERP amplitudes were more negative than blurry ERP amplitudes. Furthermore, these perceptual effects were independent from stimulus repetition (memory) because similar behavioral and ERP patterns were observed for both studied and unstudied items (Fig. 4). These results provide strong evidence that manipulating the perceptual context by altering the sequence of probe clarity (blocked or randomized sequences) affects both the mere-exposure effect and physiological differences between clear and blurry products.

The findings from the present studies, along with the behavioral evidence that social-cognitive factors such as threat (Crisp, Hutter, \& Young, 2009) and list composition (Dechêne et al., 2009) can reverse the mere exposure effect, offer strong support for the argument that the top-down interpretation of perceptual fluency drives affective judgments in certain contexts. Collectively, these effects do not support arguments that contend mere exposure phenomena is due to bottom-up perceptual processing (Zajonc, 2001) or implicit priming (Ladd et al., 2014). Those accounts predict that the same behavioral and ERP patterns would be observed across experiments, and we report stark differences across experiments that utilized identical physical stimuli. Consequently, these effects provide strong evidence that mere-exposure effects are produced by interpretations of perceptual fluency within a broad decision-making context (Leynes \& Zish, 2012; Whittlesea \& Williams, 2001a, b).

How can these findings be reconciled with the compelling evidence that affective responses are independent of cognitive processes (e.g., Ladd et al., 2014; Zajonc, 2001)? While this issue will need to be vetted in future studies that are specifically designed to address it, we speculate on one possibility to begin such an inquiry. Conscious awareness has been linked to the size of the mere exposure effect (e.g., Bornstein \& D'Agostino, 1992; Zajonc, 2001), and it is reasonable to speculate that perceptual features might interact with awareness (e.g., Willems \& Van der Linden, 2006). Consequently, changes in awareness could also possibly influence the balance of implicit and explicit memory, or affect interpretations of the perceptual fluency (Whittlesea \& Price, 2001). At the very least, this is reason to suspect that some contexts might draw more upon bottom-up processes, whereas other contexts might draw more upon interpretative processes that operate with more top-down control. We believe that ERPs are a powerful tool to provide insight into these continuing issues and will be valuable in providing physiological leverage to understanding such behavioral effects in future studies.

\section{Broader implications for understanding mechanisms of fluency}

The behavioral and physiological evidence (ERPs) here provide important new insight into fluency and its role in affective judgments. First, fluency altered the affective judgment without altering the recognition judgment. Because the affective judgment came after the recognition judgment, these unique results provide evidence that fluency can be robust enough to persist into a second judgment. ${ }^{3}$ Second, the different effects produced by the trial sequence (blocked or random) add to the extant literature indicating trial-by-trial fluctuations in fluency affects recognition (Bruett \& Leynes, 2015; Leynes $\&$ Zish, 2012). This evidence is consistent with models that argue processing discrepancies are interpreted via top-down control processing (Whittlesea \& Williams, 2001a, b). In other words, fluency is interpreted relative to the decision-making context via a dynamic process (i.e., "norms on the fly", Whittlesea \& Leboe, 2003) rather than merely being inherent to the stimulus. A big part of any task context is what happened a few seconds or minutes before the current item's evaluation (see also Addante, de Chastelaine, \& Rugg, 2015; Addante, Watrous, Yonelinas, Ekstrom, \& Ranganath, 2011; Gruber \& Otten, 2010). In Experiment 1, blocking the perceptual clarity of novel products was expected to create an opportunity for repetition fluency to support recognition and affective judgments because the rapid study exposure left behind very little memory trace information (cf., Bruett \& Leynes, 2015). On the other hand, mixing the presentation of clear and blurry novel products (Exp. 2) creates a different context such that multiple sources of fluency are varying across trials (i.e., both repetition fluency and probe clarity). These fluctuations will increase the processing discrepancies between

\footnotetext{
${ }^{3}$ We thank Marianne Lloyd for drawing our attention to this aspect of our findings.
} 
expectations and actual experiences that demand an interpretation as they happen (Whittlesea \& Williams, 2001a, 2001b). Sometimes these discrepancies will lead to perceptions of familiarity (e.g., Bruett \& Leynes, 2015) and sometimes these discrepancies will influence affective judgments (Whittlesea $\&$ Price, 2001). The results of Experiment 2 provide important physiological evidence supporting this argument because varying the pre-trial context differentially influenced the pattern of ERP results (cf., Bruett \& Leynes, 2015).

\section{Conclusions}

The data presented in this article represent a clear case where the previous trial's perceptual residue interacts with the perceptual and memorial information activated by the probe. Therefore, the results demonstrate that cognition and perception interact in some contexts to influence other cognitive judgments (affective judgments in these particular experiments). This top-down modulation of perceptual processing also highlights a serious issue with the common practice of equating early ERP differences with strictly perceptual (or automatic) processes. For example, early ERP components vary during both gender and race processing, and these early components have been automatically equated with "implicit" and "automatic" processes, respectively (Kubota \& Ito, 2009). Such conclusions must be supported by a variety of convergent evidence - not just ERPs - because the results of the present study demonstrate that top-down processes can modulate early, perceptual ERPs. These conclusions are echoed in literature demonstrating that attention can also provide topdown modulation of early ERP components (e.g., Bengson, Lopez-Calderon, \& Mangun, 2012; Luck, Woodman, \& Vogel, 2000). The present findings also suggest that social categorization judgments (e.g., group membership) that could draw on fluency are subject to some forms of top-down processing (see also Zheng \& Segalowitz, 2014).

Author Notes The authors thank Kevin Alexander, Kim Bernstein, Liz Blaskovich, Ryan Brzycki, Alexa Bolognese, Daniel Carlin, Caitlin Connolly, Jonathan Daniels, Corinne Fircak, Alyssa Fountain, Bhavika Kakadia, Rabia Khawaja, Rebecca Ruiz, Zachary Schluger, Anjani Singh, Ruhi Srinivasan, Sean Sussman, and Elizabeth Taranto for their help with collecting the data. We thank Marianne Lloyd, Jason Chein, and two anonymous reviewers for their helpful comments on an earlier draft of this paper.

\section{References}

Addante, R. J. (2015). A critical role of the human hippocampus in an electrophysiological measure of implicit memory. NeuroImage, 109, $515-528$.
Addante, R. J., de Chastelaine, M., \& Rugg, M. D. (2015). Pre-stimulus neural activity predicts successful encoding of inter-item associations. NeuroImage, 105, 21-31.

Addante, R. J., Ranganath, C., \& Yonelinas, A. P. (2012). Examining ERP correlates of recognition memory: Evidence of accurate source recognition without recollection. NeuroImage, 62, 439-450.

Addante, R. J., Watrous, A. J., Yonelinas, A. P., Ekstrom, A. D., \& Ranganath, C. (2011). Pre-stimulus theta activity predicts correct source memory retrieval. Proceedings of the National Academy of Sciences of the United States of America, 108, 10702-10707.

Bengson, J. J., Lopez-Calderon, J., \& Mangun, G. R. (2012). The spotlight of attention illuminates failed feature-based expectancies. Psychophysiology, 49, 1101-1108.

Bornstein, R. F. (1989). Exposure and affect: Overview and meta-analysis of research, 1968-1987. Psychological Bulletin, 106, 265-289.

Bornstein, R. F., \& D'Agostino, P. R. (1992). Stimulus recognition and the mere exposure effect. Journal of Personality and Social Psychology, 63, 545-552.

Bruett, H., \& Leynes, P. A. (2015). Event-related potentials indicate that fluency can be interpreted as familiarity. Neuropsychologia, 78, 41-50.

Cleary, A. M., Morris, A. L., \& Langley, M. M. (2007). Recognition memory for novel stimuli: The structural regularity hypothesis. Journal of Experimental Psychology: Learning, Memory, and Cognition, 33, 379-393.

Crisp, R. J., Hutter, R. R. C., \& Young, B. (2009). When mere exposure leads to less liking: The incremental threat effect in intergroup contexts. British Journal of Psychology, 100, 133-149.

Curran, T. (2000). Brain potentials of recollection and familiarity. Memory \& Cognition, 28(6), 923-938.

Dechêne, A., Stahl, C., Hansen, J., \& Wänke, M. (2009). Mix me a list: Context moderates the truth effect and the mere-exposure effect. Journal of Experimental Social Psychology, 45, 1117-1122.

Gruber, M. J., \& Otten, L. J. (2010). Voluntary control over prestimulus activity related to encoding. Journal of Neuroscience, 30, 9793 9800

Jacoby, L. L., \& Dallas, M. (1981). On the relationship between autobiographical memory and perceptual learning. Journal of Experimental Psychology: General, 110, 306-340.

Jacoby, L. L., Kelley, C., Brown, J., \& Jasechko, J. (1989). Becoming famous overnight: Limits on the ability to avoid unconscious influences of the past. Journal of Personality and Social Psychology, 56, 326-338.

Karpicke, J. D., McCabe, D. P., \& Roediger, H. L. (2008). False memories are not surprising: The subjective experience of an associative memory illusion. Journal of Memory and Language, 58, 10651079.

Kubota, J. T., \& Ito, T. A. (2009). You were always on my mind: How event-related potentials inform impression formation research. In T. D. Nelson (Ed.), Handbook of prejudice, stereotyping, and discrimination (pp. 333-345). New York: Taylor \& Francis.

Ladd, S. L., Toscano, W. B., Cowings, P. S., \& Gabrieli, J. D. E. (2014). Cardiovascular change during encoding predicts the nonconscious mere exposure effect. American Journal of Psychology, 127, 157182.

Leynes, A. P., \& Zish, K. (2012). Event-related potential (ERP) evidence for fluency-based recognition memory. Neuropsychologia, 50, $3240-3249$.

Lloyd, M. E., Westerman, D. L., \& Miller, J. K. (2003). The fluency heuristic in recognition memory: The effect of repetition. Journal of Memory and Language, 48, 603-614.

Luck, S. J., Woodman, G. F., \& Vogel, E. K. (2000). Event-related potential studies of attention. Trends in Cognitive Sciences, 4, 432440.

Morgenstern, M., Isensee, B., \& Hanewinkel, R. (2013). Seeing and liking cigarette advertisements: Is there a 'mere exposure' effect? European Addiction Research, 19(1), 42-46. 
Mueller, M. L., Dunlosky, J., Tauber, S. K., \& Rhodes, M. G. (2014). The font-size effect on judgments of learning: Does it exemplify fluency effects or reflect people's beliefs about memory? Journal of Memory and Language, 70, 1-12.

Mueller, M. L., Tauber, S. K., \& Dunlosky, J. (2013). Contributions of beliefs and processing fluency to the effect of relatedness on judgments of learning. Psychonomic Bulletin and Review, 20, 378-384.

Nessler, D., Mecklinger, A., \& Penney, T. (2005). Perceptual fluency, semantic familiarity and recognition-related familiarity: An electrophysiological exploration. Cognitive Brain Research, 22, 265-288.

Parks, C. M., \& Yonelinas, A. P. (2007). Moving beyond pure signaldetection models: Comment on Wixted (2007). Psychological Review, 114, 188-202.

Reber, R., \& Schwarz, N. (1999). Effects of perceptual fluency on judgments of truth. Consciousness and Cognition, 8, 338-342.

Reber, R., Schwarz, N., \& Winkielman, P. (2004). Processing fluency and aesthetic pleasure: Is beauty in the perceiver's processing experience? Personality and Social Psychology Review, 8, 364-382.

Reber, R., Winkielman, P., \& Schwarz, N. (1998). Effects of perceptual fluency of affective judgments. Psychological Science, 9(1), 45-48.

Rhodes, M. G., \& Castel, A. D. (2008). Memory predictions are influenced by perceptual information: Evidence for metacognitive illusions. Journal of Experimental Psychology: General, 137, 615-625.

Rugg, M. D., \& Coles, M. G. H. (1995). The ERP and cognitive psychology: Conceptual issues. In M. D. Rugg \& M. G. H. Coles (Eds.), Electrophysiology of mind: Event-related brain potentials and cognition. Oxford: Oxford University Press.

Rugg, M. D., \& Curran, T. (2007). Event-related potentials and recognition memory. Trends in Cognitive Science, 11, 251-257.

Ryals, A. J., Yadon, C. A., Nomi, J. S., \& Cleary, A. M. (2011). When word identification fails: ERP correlates of recognition without identification and of word identification failure. Neuropsychologia, 49, 3224-3237.

Semlitsch, H. V., Anderer, P., Schuster, P., \& Presslich, O. (1986). A solution for reliable and valid reduction of ocular artifacts, applied to the P300 ERP. Psychophysiology, 23, 696-703.

Tom, G., Nelson, C., Srzentic, T., \& King, R. (2007). Mere exposure and the endowment effect on consumer decision making. The Journal of Psychology, 141(2), 117-125.

Voss, J. L., \& Paller, K. A. (2009). An electrophysiological signature of unconscious recognition memory. Nature Neuroscience, 30, 91819188.

Voss, J. L., \& Paller, K. A. (2010). Real-time neural signals of perceptual priming with unfamiliar geometric shapes. Journal of Neuroscience, 12, 349-355.
Wang, W., Li, B., Gao, C., Xiao, X., \& Guo, C. (2015). Electrophysiological correlates associated with contributions of perceptual and conceptual fluency to familiarity. Frontiers in Neuroscience, 9, 321.

Westerman, D. L. (2008). Relative fluency and illusions of recognition memory. Psychonomic Bulletin and Review, 15, 1196-1200.

Westerman, D. L., Lloyd, M. E., \& Miller, J. K. (2002). The attribution of perceptual fluency in recognition memory: The role of expectation. Journal of Memory and Language, 47, 607-617.

Westerman, D. L., Miller, J. K., \& Lloyd, M. E. (2003). Change in perceptual form attenuates the use of the fluency heuristic in recognition. Memory \& Cognition, 31, 619-629.

Whittlesea, B. W., Jacoby, L. L., \& Girard, K. (1990). Illusions of immediate memory: Evidence of an attributional basis for feelings of familiarity and perceptual quality. Journal of Memory and Language, 29, 716-732.

Whittlesea, B. W. A., \& Leboe, J. P. (2003). Two fluency heuristics (and how to tell them apart) basis. Journal of Memory and Language, 49, 62-79.

Whittlesea, B. W. A., \& Price, J. R. (2001). Implicit/explicit memory versus analytic/nonanalytic processing: Rethinking the mere exposure effect. Memory \& Cognition, 29, 234-246.

Whittlesea, B. W. A., \& Williams, L. (2001a). The discrepancy attribution hypothesis: I. The heuristic basis of feelings of familiarity. Journal of Experimental Psychology: Learning, Memory, and Cognition, 27, $3-13$.

Whittlesea, B. W. A., \& Williams, L. (2001b). The discrepancy attribution hypothesis: II. Expectation, uncertainty, surprise and feelings of familiarity. Journal of Experimental Psychology: Learning, Memory, and Cognition, 27, 14-33.

Willems, S., \& Van der Linden, M. (2006). Mere exposure effect: A consequence of direct and indirect fluency-preference links. Consciousness and Cognition, 15, 323-341.

Wixted, J. T. (2007). Dual-process theory and signal-detection theory of recognition memory. Psychological Review, 114, 152-176.

Zajonc, R. B. (1968). Feeling and thinking: Preferences need no inferences. American Psychologist, 35, 117-123.

Zajonc, R. B. (2001). Mere exposure: A gateway to the subliminal. Current Directions in Psychological Science, 10, 224-228.

Zebrowitz, L. A., White, B., \& Wieneke, K. (2008). Mere exposure and racial prejudice: Exposure to other-race faces increases liking for strangers of that race. Social Cognition, 26, 259-275.

Zheng, X., \& Segalowitz, S. J. (2014). Putting a face in its place: In- and out-group membership alters the N170 response. Social Cognitive and Affective Neuroscience, 9, 961-968. 